\title{
Traditional Chinese and western medicine for the prevention of deep venous thrombosis after lower extremity orthopedic surgery: a meta-analysis of randomized controlled trials
}

\author{
Shibai Zhu' ${ }^{1}$ Yi Song ${ }^{2}, X_{i}$ Chen $^{1}$ and Wenwei Qian ${ }^{1 *}$
}

\begin{abstract}
Background: Chinese herbal medicine has traditionally been considered to promote blood circulation to remove obstruction in the channels and clear pathogenic heat to drain dampness effects. We conducted this meta-analysis to evaluate its benefits for the prevention of deep venous thrombosis (DVT) after lower extremity orthopedic surgery.

Methods: Relevant, published studies were identified using the following keywords: lower extremity orthopedic surgery, arthroplasty, joint replacement, fracture, traditional Chinese and western medicine, Chinese herbal medicine, deep venous thrombosis (DVT), and Venous thromboembolism (VTE). The following databases were used to identify the literature consisting of RCTs with a date of search of 31 May 2017: PubMed, Cochrane Library, Web of knowledge, the Chinese National Knowledge Infrastructure Database, the Chongqing VIP Database, the Chinese Biomedical Database, and the Wanfang Database (including three English and four Chinese databases). All relevant data were collected from studies meeting the inclusion criteria. The outcome variables were the incidence rate of DVT, activated partial thromboplastin time (APT), prothrombin time (PT), and D-dimer; subcutaneous hematoma; and other reported outcomes. RevMan5.2. software was adopted for the meta-analysis.
\end{abstract}

Results: A total of 20 published studies (1862 cases) met the inclusion criteria. The experimental group, 910 patients (48.87\%), received the Chinese herbal medicine or traditional Chinese and western medicine for prevention of DVT; the control group, 952 patients (51.13\%), received the standard western treatment. The meta-analysis showed that traditional Chinese and western medicine therapy reduced the incidence rates of DVT significantly when compared with controls (risk ratio $[R R]=0.40 ; 95 \% \mathrm{Cl}, 0.30$ to $0.54 ; P<0.00001$ ), and the D-dimer was lower in the experimental group $(P=0.01)$. Besides, the incidence rate of subcutaneous hematoma was lower in the experimental group $(P<0.0001)$. However, no significant difference was found in the PT $(P=0.98)$ and APTT $(P=0.75)$ in two groups. No serious adverse events were reported.

Conclusion: Traditional Chinese and western medicine therapy may be a safe, effective prevention modality for DVT after lower extremity orthopedic surgery. Further rigorously designed, randomized trials are warranted.

Keywords: Lower extremity orthopedic surgery, Deep venous thrombosis (DVT), Traditional Chinese and western medicine, Meta-analysis

\footnotetext{
*Correspondence: qianww007@163.com

${ }^{1}$ Department of Orthopedic Surgery, Peking Union Medical College Hospital,

Peking Union Medical College, Chinese Academy of Medical Science, Beijing,

China

Full list of author information is available at the end of the article
} 


\section{Background}

Deep vein thrombosis (DVT) is one of the most common complications after lower extremity orthopedic surgery, several patients may develop into pulmonary embolism (PE), and some serious can lead to death [1, 2]. A large number of researches show that patients undergoing lower extremity orthopedic surgery are the high-risk group for venous thromboembolism (VTE). In the absence of any preventive measures, the incidence of DVT after surgery was up to $40-60 \%$, the incidence of PE was 20\%, and the fatal PE was $0.1-2 \%$ [1]. Thus, guidelines for the prevention of postoperative anticoagulation have been recommended in various countries after excluding the contraindication [1-6].

Traditional Chinese medicine theory holds that DVT is the category of "pulse closed" and "femoral swelling," and the main treatment is to promote blood circulation to remove blood stasis. Traditional Chinese herb not only has the effects of analgesic and anti-inflammatory, but also can effectively improve blood circulation [7]. Thus, in conducting the traditional Chinese medicine syndrome differentiation, adjuvant therapy on the basis of western medicine treatment is paying more attention to the theory of traditional Chinese medicine that diagnosis and treatment are based on an overall analysis of the illness and the patient's condition and also insisting on treating both principal and secondary aspect of disease. Therefore, the advantages of the combination of traditional Chinese and western medicine are becoming more and more prominent in the treatment and prevention of thrombotic diseases.

Although there is a long history of traditional Chinese medicine for prevention and treatment of DVT in ancient China, the necessary quantitative evidence to estimate treatment effects is still lacking. Therefore, we conducted this meta-analysis to evaluate the efficacy and safety of RCTs involving traditional Chinese and western medicine for the prevention of DVT after lower extremity orthopedic surgery, expecting to provide evidencebased medical proof for clinical medicine.

\section{Methods}

\section{Search strategy}

A comprehensive search for studies about traditional Chinese and western medicine for the prevention of DVT after lower extremity orthopedic surgery was conducted through the online database. We searched PubMed, Cochrane Library, Web of knowledge, the Chinese National Knowledge Infrastructure Database, the Chongqing VIP Database, the Chinese Biomedical Database, and the Wanfang Database (including three English and four Chinese databases) up to May 31, 2017. The following keywords were used: ("traditional Chinese and western medicine" or "traditional Chinese medicine" or "Chinese herbal medicine" or "Chinese herb") in combination with (("lower extremity orthopedic surgery" or "arthroplasty" or "joint replacement" or "fracture") and ("deep venous thrombosis" or "Venous thromboembolism")). Additional studies were identified from references of retrieved articles. The duplicated articles were eliminated using Endnote software (EndNote X7).

\section{Inclusion and exclusion criteria}

Trials were eligible if they were randomized controlled trials (RCTs) recruiting participants with lower extremity orthopedic surgery (including total hip replacement [THR], total knee replacement [TKR], or hip fracture surgery [HFS]) and so on). These included patients in the experimental group who received the treatment of traditional Chinese and western medicine for the prevention of DVT after orthopedic surgery, while those in the control group were subjected to standard western therapy for DVT. All studies cited could provide relevant data. There was no language restriction in the literature search.

In order to evaluate the independent effects of the traditional Chinese and western medicine intervention, we excluded (1) conference abstracts, review articles, animal studies, cadaveric studies, in vitro studies, or articles published in a form other than clinical trials; (2) any control group that included traditional Chinese therapies; (3) literatures without relevant postoperative indicators or quantitative data; and (4) repeated published literature.

\section{Selection of studies}

Two authors (Zhu and Song) independently screened all potential eligible studies. Titles and abstracts were first screened to exclude irrelevant citations. Full text of all articles of potentially relevant abstracts were retrieved and screened according to the study inclusion and exclusion criteria.

\section{Data extraction}

The two investigators (Zhu and Qian) reviewed the titles and abstracts, carefully read the full texts according to preset inclusion criteria, and extracted the data from included studies using a pre-designed data extraction table. Study characteristics that were extracted included the author, publication year, sample size, age and gender of subjects, and detailed information of two groups, outcome measures, summary of results, main conclusion, and adverse reactions. The data were arranged into experimental form and Excel spreadsheets in duplicate. All data extraction work was done by the two authors independently. When any inconsistency arose, the issues were either resolved by a third investigator (Chen) or negotiated by both the original investigators. 


\section{Definition of outcome events}

The main outcome events were the incidence of DVT, the outcomes of D-dimer, prothrombin time (PT), and thromboplastin time (APTT). The secondary events were any adverse events (including hematoma, hemorrhage, and so on) and other reported outcomes.

\section{Quality assessment}

Two reviewers (Zhu and Song) independently conducted the methodological quality of all included studies. The cases were reviewed and screened carefully for data of interest. Any disagreement between the investigators was resolved with mutual consensus in the presence of the third author (Chen). The quality of studies was estimated according to the Newcastle-Ottawa Scale (NOS) [8].

\section{Statistical method}

For each included study, the weighted mean differences (WMD) at 95\% confidence intervals (CI) were calculated for continuous outcomes, while odds ratio (OR) at 95\% confidence intervals $(\mathrm{CI})$ were calculated for dichotomous outcomes. Heterogeneity among the studies was assessed using the chi-squared and $I$-squared $\left(I^{2}\right)$ tests. A fixed effect model was applied when $I^{2}<50 \%$, whereas a random effect model was applied when $I^{2}>50 \%$. All analyses were completed with Review Manager 5.2 [9, 10] software (Cochrane Collaboration, Oxford, UK) and a $P$ value $<0.05$ was considered statistically significant. Funnel plots were used to assess potential bias [11].

\section{Results}

The flow of study identification and inclusion are shown in Fig. 1. In summary, a total of 520 abstracts identified from online databases (including three English and four Chinese databases). After initially screening 129 potentially relevant abstracts, we excluded 75 because they did not meet the inclusion criteria (reviews, case reports, or duplicate publications). We retrieved and reviewed 54 full articles; 34 were excluded due to lack of randomization or absence of a control group $(n=15)$, major methodologic flaws, and insufficient data $(n=19)$. Finally, 20 eligible RCTs $[12-31]$ involving 1862 patients were included.

\section{Basic characteristics of included studies}

The characteristics of the 20 RCTs are summarized in Tables 1, 2, 3, 4, 5, and 6. All 20 RCTs were conducted in China and were published between 2006 and 2017. There are a total of 910 participants who received traditional Chinese and western medicine (experimental group) and 952 who received standard western treatment (control group). All 20 papers reported the incidence rate of DVT after lower extremity orthopedic surgery, 9 papers had data on D-dimer, 8 papers had the outcomes of PT and APTT, and 4 papers reported subcutaneous hematoma. All papers had similar distributions of sex, age, and types of surgery. The included studies are described in Table 1 . Table 2 includes the composition and efficacy of traditional Chinese medicine prescription in the experimental groups. The incidence rate of DVT are listed in Table 3, the data of APTT and PT are in Table 4, and the outcome of D-dimer is described in Table 5. The date of subcutaneous hematoma is summarized in Table 6.

\section{Results of the meta-analysis}

In the eligible RCTs, all trials measured the incidence rate of DVT [12-31], while 8 trials reported the change of PT and APTT [16, 19, 23-26, 30, 31]. And 9 trials focused on the data of D-dimer $[12,16,19,21,23,24,26$,

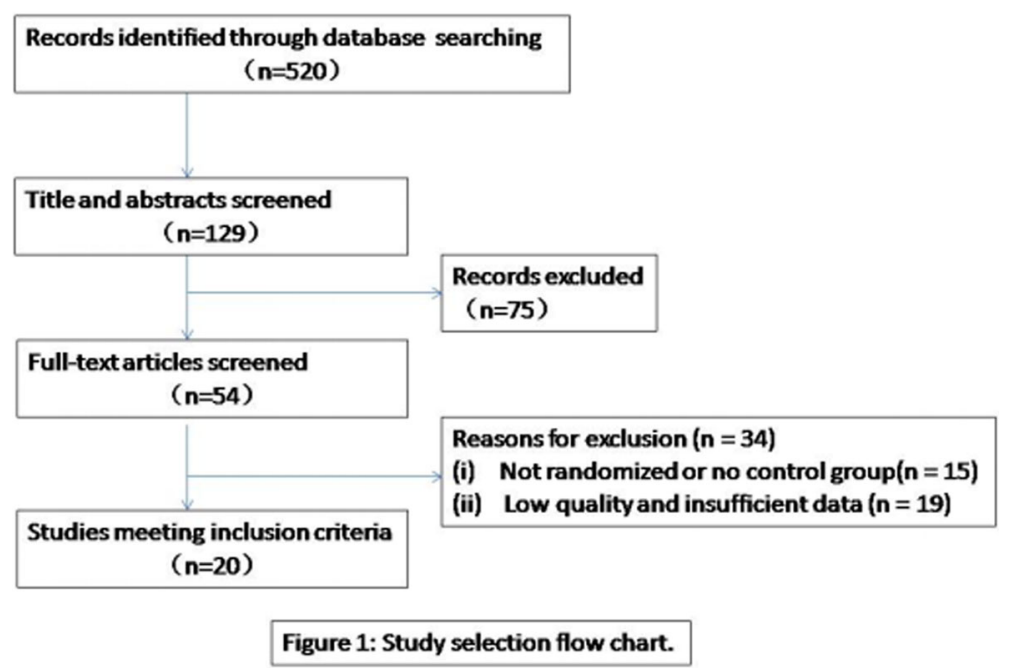

Fig. 1 Flow diagram of searches 


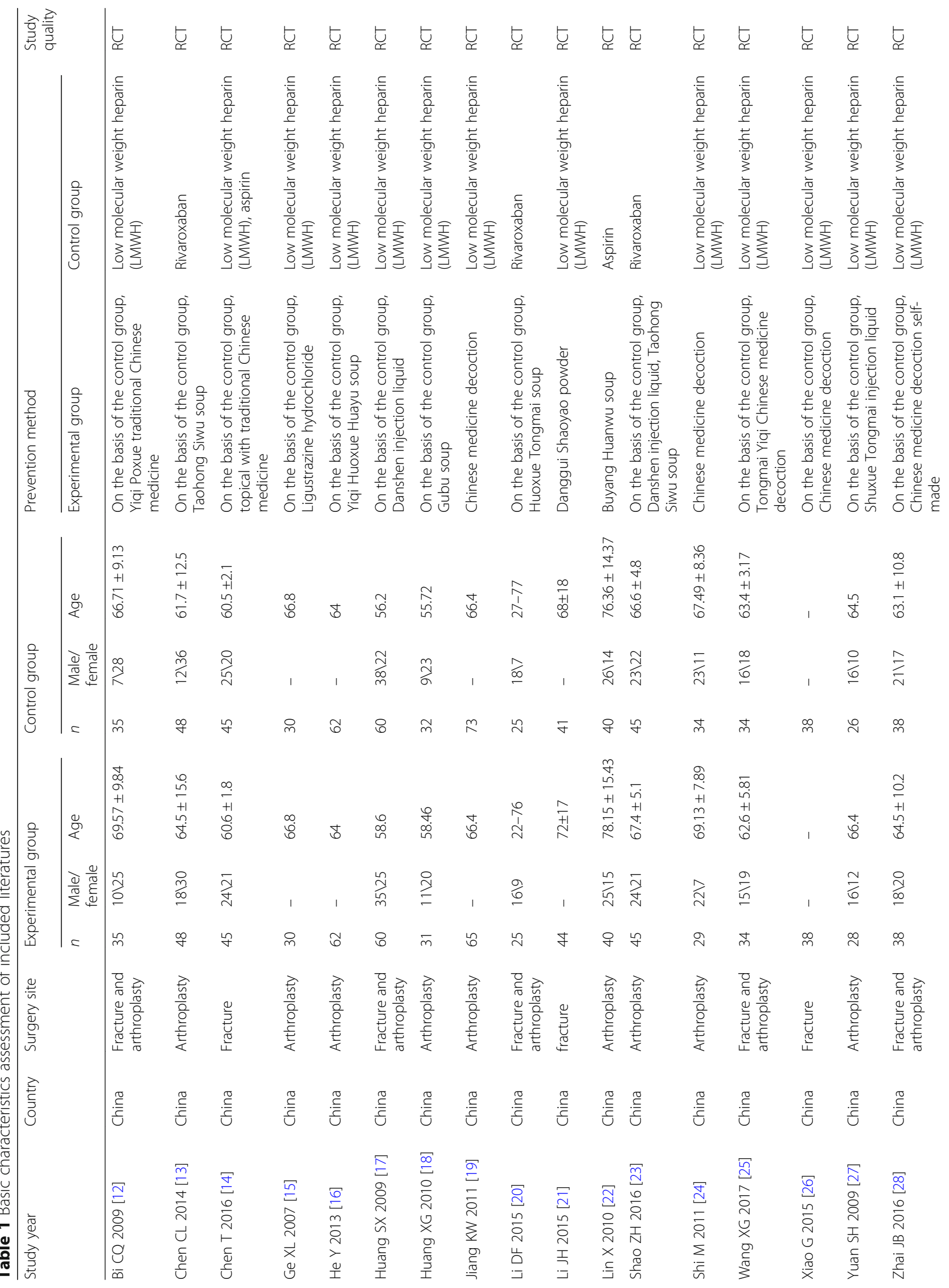




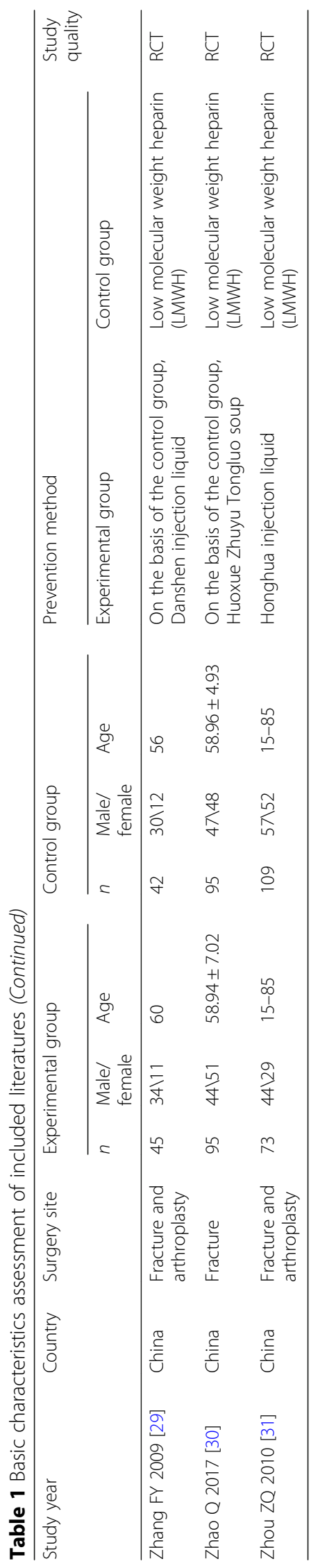


Table 2 The composition and efficacy of traditional Chinese medicine prescription in the experimental groups

\begin{tabular}{|c|c|c|}
\hline First author and year & Traditional Chinese medicine compound & Treatment time \\
\hline Bi CQ 2009 [12] & $\begin{array}{l}\text { Huangqi } 60 \mathrm{~g} \text {, shuizhi } 5 \mathrm{~g} \text {, gancao } 5 \mathrm{~g} \text {, sanqi } \\
3 \mathrm{~g}\end{array}$ & $\begin{array}{l}\text { Water decoction, po, bid, } \\
7 \text { days }\end{array}$ \\
\hline Chen CL 2014 [13] & $\begin{array}{l}\text { Taohong siwu soup:taoren } 10 \mathrm{~g} \text {, honghua } \\
10 \mathrm{~g} \text {, danggui } 12 \mathrm{~g} \text {, chishuo } 10 \mathrm{~g} \text {, chuangxiong } \\
6 \mathrm{~g} \text {, dihuang } 15 \mathrm{~g} \text {, chuangshanjia } 6 \mathrm{~g} \text {, chaihu } \\
12 \mathrm{~g} \text {, huangqi } 10 \mathrm{~g} \text {, xiangfu } 12 \mathrm{~g} \text {, yanhusuo } \\
15 \mathrm{~g} \text {, xuduan } 15 \mathrm{~g} \text {, gancao } 6 \mathrm{~g}\end{array}$ & $\begin{array}{l}\text { Water decoction, po, bid, } \\
7 \text { days }\end{array}$ \\
\hline
\end{tabular}

Chen T 2016 [14] Huajiao, sumu, mugua, shenjincao, danggui, niuxi

Ge XL 2007 [15] Yansuan chuanxiong injection 80 mg

He Y 2013 [16]

Huang SX 2009 [17]

Huang XG 2010 [18] Gubu soup:huangqi $30 \mathrm{~g}$, dangshen $30 \mathrm{~g}$, jixueteng $30 \mathrm{~g}$, danggui $15 \mathrm{~g}$, danshen $30 \mathrm{~g}$, chishuo $15 \mathrm{~g}$, niuxi $15 \mathrm{~g}$, baishu $15 \mathrm{~g}$, yiren $20 \mathrm{~g}$, huangbai $20 \mathrm{~g}$, gancao $10 \mathrm{~g}$

Jiang KW 2011 [19] Huangqi $30 \mathrm{~g}$, shuizhi $3 \mathrm{~g}$, sanqi $3 \mathrm{~g}$

Li DF 2015 [20]

Li JH 2015 [21]

Lin X 2010 [22]

Shao ZH 2016 [23]

Shi M 2011 [24]

Wang XG 2017 [25]

Xiao G 2015 [26]

Yuan SH 2009 [27]

Zhai JB 2016 [28]

Zhang FY 2009 [29] $10 \mathrm{~g}$, baishu $10 \mathrm{~g}$, gancao $10 \mathrm{~g}$, honghua $10 \mathrm{~g}$, danggui $10 \mathrm{~g}$, huangqi $10 \mathrm{~g}$, taoren $10 \mathrm{~g}$, shudi $6 \mathrm{~g}$, chuanxiong $6 \mathrm{~g}$, chishuo $6 \mathrm{~g}$, zhike $6 \mathrm{~g}$, jiegeng $6 \mathrm{~g}$, dilong $3 \mathrm{~g}$

Danshen injection $20 \mathrm{ml}$ and physiological Danshen injectio

Huoxue tongmai soup:shuizhi $5 \mathrm{~g}$, honghua $10 \mathrm{~g}$, danggui $10 \mathrm{~g}$, huangqi $20 \mathrm{~g}$, danshen $10 \mathrm{~g}$, fuling $10 \mathrm{~g}$, yanhusuo $8 \mathrm{~g}$, sanqi $6 \mathrm{~g}$

Danggui shuoyao pills: danggui $3 \mathrm{~g}$, chishuo $16 \mathrm{~g}$, chuanxiong $8 \mathrm{~g}$, fuling $4 \mathrm{~g}$, zhexie $8 \mathrm{~g}$, baishu $4 \mathrm{~g}$ dangguiwei $6 \mathrm{~g}$, taoren $6 \mathrm{~g}$, honghua $6 \mathrm{~g}$, chuanxiong $9 \mathrm{~g}$, chishuo $9 \mathrm{~g}$, dilong $3 \mathrm{~g}$, huainiuxi $9 \mathrm{~g}$

Danshen injection $20 \mathrm{ml}$, and 5\% glucose injection; taohong siwu soup:honghua $10 \mathrm{~g}$, taoren $15 \mathrm{~g}$, danggui $15 \mathrm{~g}$, chuanxiong $15 \mathrm{~g}$, chishuoyao $15 \mathrm{~g}$, niuxi $15 \mathrm{~g}$, yinhuateng $20 \mathrm{~g}$, yirenren $15 \mathrm{~g}$, baishu $15 \mathrm{~g}$, fuling $15 \mathrm{~g}$, zhichuanwu $15 \mathrm{~g}$, zhiruxiang $15 \mathrm{~g}$

Shenghuangqi, dangguiwei, chishuo, dilong, chuanxiong, taoren, honghua

Tongmaiyiqi soup:huangqi $15 \mathrm{~g}$, baishu $15 \mathrm{~g}$,

Huoxue tongmai soup:huangqi $30 \mathrm{~g}$, niuxi $15 \mathrm{~g}$, taoren $15 \mathrm{~g}$, danshen $15 \mathrm{~g}$, chishuo $15 \mathrm{~g}$, shudi $15 \mathrm{~g}$, chuanxiong $10 \mathrm{~g}$, honghua $10 \mathrm{~g}$, dangguiwei $10 \mathrm{~g}$, shuizhi $5 \mathrm{~g}$

Shuxuetong injection $6 \mathrm{ml}$ and $0.9 \%$ saline $250 \mathrm{ml}$

Chuanxiong $10 \mathrm{~g}$, shuizhi $5 \mathrm{~g}$, honghua $15 \mathrm{~g}$, dangshen $10 \mathrm{~g}$, danggui $5 \mathrm{~g}$, danshen $15 \mathrm{~g}$, niuxi $15 \mathrm{~g}$

Danshen injection $20 \mathrm{ml}$
Yiqi huoxue huayu soup:dangshen $10 \mathrm{~g}$, fuling Water decoction, po, bid, po,

Buyang huanwu soup:sheng huangqi $30 \mathrm{~g}$, quanxie $6 \mathrm{~g}$, sanqi $6 \mathrm{~g}$, shuizhi $6 \mathrm{~g}$, zhexie $9 \mathrm{~g}$
Water decoction, hot pack, bid ivgit, qd, 10 days

21 days

ivgit, qd, 7 days

Water decoction, po, bid, po, 9 days

Water decoction, po, bid, po, 7 days

Water decoction, po, bid, po, 7 days

Water pills, 5 g/every time, po, qd, 7 days

Water decoction, po, bid, po, 7 days

Danshen injection:ivgit, qd; taohong siwu soup: water decoction, po, bid

Water decoction, po, bid, po, 7 days

Water decoction, po, bid, po, 14 days

Water decoction, po, bid, po, 14 days

ivgit, qd, 15 days

Water decoction, po, bid, po, 14 days

IV, qd, 7 days

\section{Effect}

Promoting blood circulation to remove obstruction in the channels

Promoting blood by nourishing the blood

Promoting blood circulation to relieve pain Antiplatelet agglutination, promoting blood circulation to remove obstruction in the channels

Promoting blood circulation to remove obstruction in the channels

Promoting blood circulation to remove obstruction in the channels

Promoting blood circulation to remove obstruction in the channels

Promoting blood circulation to remove obstruction in the channels, pain relief by diminishing swelling

Promoting blood circulation to remove obstruction in the channels

Promoting blood by nourishing the blood, clearing heat, and removing dampness

Promoting blood circulation to remove obstruction in the channels

Promoting blood by nourishing the blood

Promoting blood circulation to remove obstruction in the channels, pain relief by diminishing swelling

Promoting blood circulation to remove obstruction in the channels

Promoting blood by nourishing the blood

Promoting blood circulation to remove obstruction in the channels

Promoting blood circulation to remove obstruction in the channels, pain relief by diminishing swelling

Promoting blood circulation to remove obstruction in the channels 
Table 2 The composition and efficacy of traditional Chinese medicine prescription in the experimental groups (Continued)

\begin{tabular}{llll}
\hline First author and year & Traditional Chinese medicine compound & Treatment time & Effect \\
\hline Zhao Q 2017 [30] & $\begin{array}{l}\text { Huoxue zhuyu tongluo soup:dangguo } 20 \mathrm{~g}, \\
\text { yirenren } 5 \mathrm{~g} \text {, danshen } 10 \mathrm{~g} \text {, shangzhi } 5 \mathrm{~g},\end{array}$ & $\begin{array}{l}\text { Water decoction, po, bid, po, } \\
\text { tubie } 5 \mathrm{~g} \text {, sumu } 10 \mathrm{~g} \text {, chuanxiong } 20 \mathrm{~g} \text {, niuxi }\end{array}$ & Promoting blood circulation to relieve pain \\
& $\begin{array}{l}\mathrm{g} \mathrm{g} \\
\text { Honghua injection } 20 \mathrm{ml} \text { and 0.9\% saline }\end{array}$ & ivgit, qd, 10 days & $\begin{array}{l}\text { Promoting blood circulation to remove } \\
\text { obstruction in the channels }\end{array}$ \\
\hline
\end{tabular}

28, 30]. Besides, 4 RCTs $[12,19-21]$ reported the incidence of subcutaneous hematoma.

\section{Comparison of the incidence of DVT between the two groups}

All 20 trials [12-31] involving 1862 patients measured the incidence rate of DVT, including 910 cases in the experimental groups and 952 cases in the control groups. There was a small degree of statistical heterogeneity across studies $\left(I^{2}=20 \%\right)$, and a fixed effects model was used for statistical analysis. The pooled analysis indicated that traditional Chinese medicine reduced the incidence rates of DVT significantly when compared with controls (risk ratio $[R R]=0.40 ; 95 \% \mathrm{CI}, 0.30$ to $0.54 ; P<0.00001$ ) (Fig. 2).

\section{PT, APTT, and D-dimer outcomes}

Eight trials [16, 19, 23-26, 30, 31] involving 931 patients measured PT and APTT, and nine trials [12, 16, 19, 21, $23,24,26,28,30]$ involving 912 patients measured Ddimer. The random effects model was used for statistical analysis. The pooled analysis indicated that statistical heterogeneity was found among studies (PT: $I^{2}=92 \%, P$ $<0.00001$; APTT: $I^{2}=98 \%, P<0.00001$; D-dimer: $I^{2}=$ $83 \%, P<0.00001)$; the results showed there are no statistical difference of PT and APTT between the two groups (PT: $\mathrm{MD}=0.01,95 \% \mathrm{CI}:-0.56$ to $0.58, P=0.98$, Fig. 3; APTT: $\mathrm{MD}=-0.71,95 \% \mathrm{CI}:-4.97$ to $3.54, P=0$. 74, Fig. 4). However, there is a statistical difference of Ddimer between the two groups ( $D$-dimer: $M D=-0.18$, 95\% CI: -0.32 to $-0.04, P=0.01$, Fig. 5 ).

Table 3 Test data source and data extraction (incidence rate of DVT)

\begin{tabular}{|c|c|c|c|c|}
\hline First author and year & DVT diagnosis & Experimental group & Control group & DVT site \\
\hline Bi CQ 2009 [12] & Color Doppler ultrasound & $0 / 35(0.00 \%)$ & $1 / 35(2.86 \%)$ & Not mentioned \\
\hline Chen CL 2014 [13] & Not mentioned & $1 / 48(2.1 \%)$ & $4 / 488.3 \%$ & Not mentioned \\
\hline Chen T 2016 [14] & Not mentioned & $2 / 45(4.44 \%)$ & $15 / 45(33.33 \%)$ & Not mentioned \\
\hline Ge XL 2007 [15] & Color Doppler ultrasound, Angiography & $0 / 30(0 \%)$ & $2 / 30(6.67 \%)$ & Distal thrombosis of the lower limbs \\
\hline He Y 2013 [16] & Color Doppler ultrasound & $5 / 62(8.1 \%)$ & $7 / 62(11.3 \%)$ & Not mentioned \\
\hline Huang SX 2009 [17] & Color Doppler ultrasound & $2 / 60(3.3 \%)$ & $12 / 60(20 \%)$ & Not mentioned \\
\hline Huang XG 2010 [18] & Color Doppler ultrasound & $4 / 31(12.9 \%)$ & $7 / 32(21.9 \%)$ & $\begin{array}{l}\text { Popliteal vein thrombosis } 3 \text { case, calf } \\
\text { venous plexus } 8 \text { case }\end{array}$ \\
\hline Jiang KW 2011 [19] & Color Doppler ultrasound, Angiography & $12 / 65(18 \%)$ & $8 / 73(11 \%)$ & $\begin{array}{l}\text { Femoral vein thrombosis } 1 \text { case, } \\
\text { popliteal vein thrombosis } 1 \text { case, } \\
\text { gastrocnemius thrombosis } 18 \text { case }\end{array}$ \\
\hline Li DF 2015 [20] & Not mentioned & $2 / 25(8.0 \%)$ & $4 / 25(16.0 \%)$ & Not mentioned \\
\hline Li JH 2015 [21] & Color Doppler ultrasound & $1 / 44(2.3 \%)$ & $6 / 41(14.6 \%)$ & Not mentioned \\
\hline Lin X 2010 [22] & Color Doppler ultrasound & $4 / 40(10.0 \%)$ & $10 / 40(25.0 \%)$ & Not mentioned \\
\hline Shao ZH 2016 [23] & Color Doppler ultrasound & $1 / 45(2.0 \%)$ & $6 / 45(13.0 \%)$ & Lower limb femoral vein \\
\hline Shi M 2011 [24] & Color Doppler ultrasound & 0/29 (0\%) & $0 / 34(0 \%)$ & Lower limb femoral vein \\
\hline Wang XG 2017 [25] & Color Doppler ultrasound & $1 / 34(2.94 \%)$ & $2 / 34(5.88 \%)$ & Not mentioned \\
\hline Xiao G 2015 [26] & Not mentioned & 0/38 (0\%) & 4/38 (10.53\%) & Not mentioned \\
\hline Yuan SH 2009 [27] & Color Doppler ultrasound & $1 / 28(3.57 \%)$ & 6/26 (23.07\%) & Not mentioned \\
\hline Zhai JB 2016 [28] & Color Doppler ultrasound & $1 / 38(2.6 \%)$ & $6 / 38(15.8 \%)$ & Not mentioned \\
\hline Zhang FY 2009 [29] & Color Doppler ultrasound & $4 / 45(8.9 \%)$ & $7 / 42(16.7 \%)$ & $\begin{array}{l}\text { Femoral vein thrombosis } 7 \text { case, } \\
\text { popliteal vein thrombosis } 4 \text { case }\end{array}$ \\
\hline Zhao Q 2017 [30] & Color Doppler ultrasound & $9 / 95(9.5 \%)$ & 26/95 (27.4\%) & Not mentioned \\
\hline Zhou ZQ 2010 [31] & Color Doppler ultrasound & $5 / 73(6.8 \%)$ & 11/109 (10.1\%) & Not mentioned \\
\hline
\end{tabular}




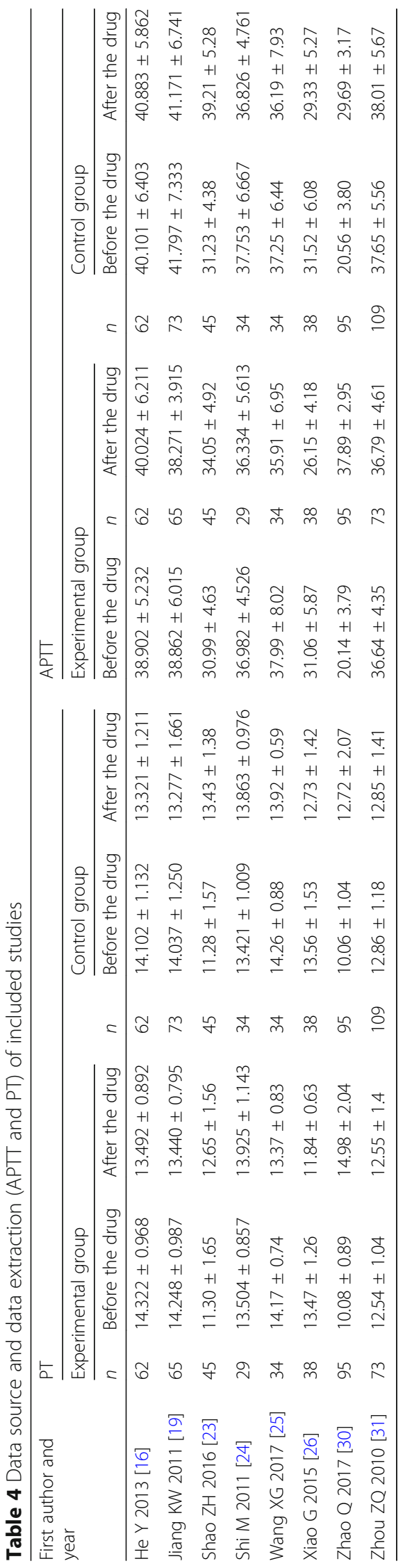


Table 5 Data source and data extraction (D-dimer) of included studies

\begin{tabular}{|c|c|c|c|c|c|c|}
\hline \multirow{2}{*}{$\begin{array}{l}\text { First author and } \\
\text { year }\end{array}$} & \multicolumn{3}{|c|}{ Experimental group } & \multirow[b]{2}{*}{$n$} & \multicolumn{2}{|l|}{ Control group } \\
\hline & Before the drug & $n$ & After the drug & & Before the drug & After the drug \\
\hline Bi CQ 2009 [12] & $1.12 \pm 1.11033$ & 35 & $0.6829 \pm 0.4547$ & 35 & $0.74 \pm 0.90365$ & $0.72 \pm 0.53292$ \\
\hline He Y 2013 [16] & $1.242 \pm 1.078$ & 62 & $0.792 \pm 0.592$ & 62 & $0.832 \pm 0.912$ & $0.792 \pm 0.612$ \\
\hline Jiang KW 2011 [19] & $1.120 \pm 1.110$ & 65 & $0.682 \pm 0.454$ & 73 & $0.740 \pm 0.903$ & $0.720 \pm 0.532$ \\
\hline Li JH 2015 [21] & $0.856 \pm 0.187$ & 44 & $0.509 \pm 0.284$ & 41 & $0.791 \pm 0.221$ & $0.693 \pm 0.457$ \\
\hline Shao ZH 2016 [23] & $0.154 \pm 0.060$ & 45 & $1.405 \pm 0.362$ & 45 & $0.142 \pm 0.051$ & $1.968 \pm 0.527$ \\
\hline Shi M 2011 [24] & $0.986 \pm 0.325$ & 29 & $0.603 \pm 0.428$ & 34 & $0.962 \pm 0.547$ & $0.891 \pm 0.734$ \\
\hline Xiao G 2015 [26] & $0.16 \pm 0.09$ & 38 & $0.11 \pm 0.26$ & 38 & $0.17 \pm 0.10$ & $0.16 \pm 0.11$ \\
\hline Zhai JB 2016 [28] & $1.525 \pm 0.347$ & 38 & $1.642 \pm 0.389$ & 38 & $1.493 \pm 0.321$ & $2.205 \pm 0.561$ \\
\hline Zhao Q 2017 [30] & $1.90 \pm 0.43$ & 95 & $1.65 \pm 0.56$ & 95 & $1.90 \pm 0.40$ & $1.62 \pm 0.62$ \\
\hline
\end{tabular}

\section{Subcutaneous hematoma}

Only 4 RCTs [12, 19-21] involving 315 patients reported the incidence rate of subcutaneous hematoma. There was no statistical heterogeneity across studies $\left(I^{2}=21 \%\right)$, and a fixed effects model was used for statistical analysis. The pooled analysis indicated a significantly lower number of patients on experimental group undergoing the subcutaneous hematoma compared to that on the control group (risk ratio $[R R]=0.35$; $95 \%$ CI, 0.22 to $0.56 ; P<0.00001$ ) (Fig. 6).

\section{Quality assessment}

The quality assessment of the trials was performed using the Newcastle-Ottawa Scale. The detailed results are presented in Fig. 7. The overall quality of trials was moderate. Randomization was adequate in 20 trials (100\%). All studies reported the similarity of study groups at baseline (100\%). Outcome assessors blinded in 2 trials (10\%), unclear in 18 trials (90\%), the bias of blinding to patients in 2 trials (10\%), unclear in 18 trials (90\%). Allocation concealment and intention to treat items were difficult to assess from reported information.

\section{Discussion}

DVT is a common complications after lower extremity orthopedic surgery, especially in THA, TKA, and hip fracture. The incidence of VTE is very high in patients

Table 6 Data source and data extraction (subcutaneous hematoma) of included studies

\begin{tabular}{lll}
\hline First author and year & \multicolumn{2}{l}{ Subcutaneous hematoma } \\
\cline { 2 - 3 } & Experimental group & Control group \\
\hline Bi CQ 2009 [12] & $10 / 35(28.57 \%)$ & $26 / 35(74.29 \%)$ \\
Jiang KW 2011 [19] & $1 / 65(2.2 \%)$ & $8 / 73(17.8 \%)$ \\
Li DF 2015 [20] & $4 / 25(8.9 \%)$ & $11 / 25(44.0 \%)$ \\
Li JH 2015 [21] & $2 / 44(4.5 \%)$ & $1 / 41(2.4 \%)$ \\
\hline
\end{tabular}

who do not receive preventive anticoagulation therapy, some serious can develop into PE. The rescue success of fatal PE is extremely low and without any aura, which is one of the most common causes of non-expected death in the hospital. Therefore, the universality of DVT and the severity of PE after lower extremity orthopedic surgery have been formed into a consensus among doctors in different countries. Furthermore, lots of studies and guidelines have demonstrated that patients received lower extremity orthopedic surgery should begin preventive anticoagulation as soon as possible after ruled out any contraindications.

At present, the mechanical preventive measures of DVT includes graduated compression stocking (GCS), intermittent pneumatic compression devices (IPC), venous foot pump (VFP), early mobilization, and so on. However, the commonly used anticoagulant drugs, such as the unfractionated and warfarin from the last century to low molecular weight heparin (LMWH), and burgeonsing direct $\mathrm{Xa}$ factor inhibitors. Besides, the latest guidelines of AAOS also explicitly recommended aspirin as a drug for the prevention of DVT after lower extremity orthopedic surgery [2].

For Chinese herbal medicine, also called traditional Chinese medicine, most western scholars may think that it is not a science, but an experience, and it is not supported by any theoretical foundation. However, in fact, using the traditional Chinese herbal medicine for prevention and treatment of thrombosis has been a thousand years of history in China; there is its own unique advantages. Promoting circulation and removing stasis and clearing heat and promoting diuresis are the main theory in the prevention and treatment of DVT [32, 33].

A total of 20 researched involving 1862 patients were included in our study; all papers are RCTs. The results of meta-analysis showed that traditional Chinese and western medicine therapy had obvious advantages in the prevention of DVT after lower extremity orthopedic 


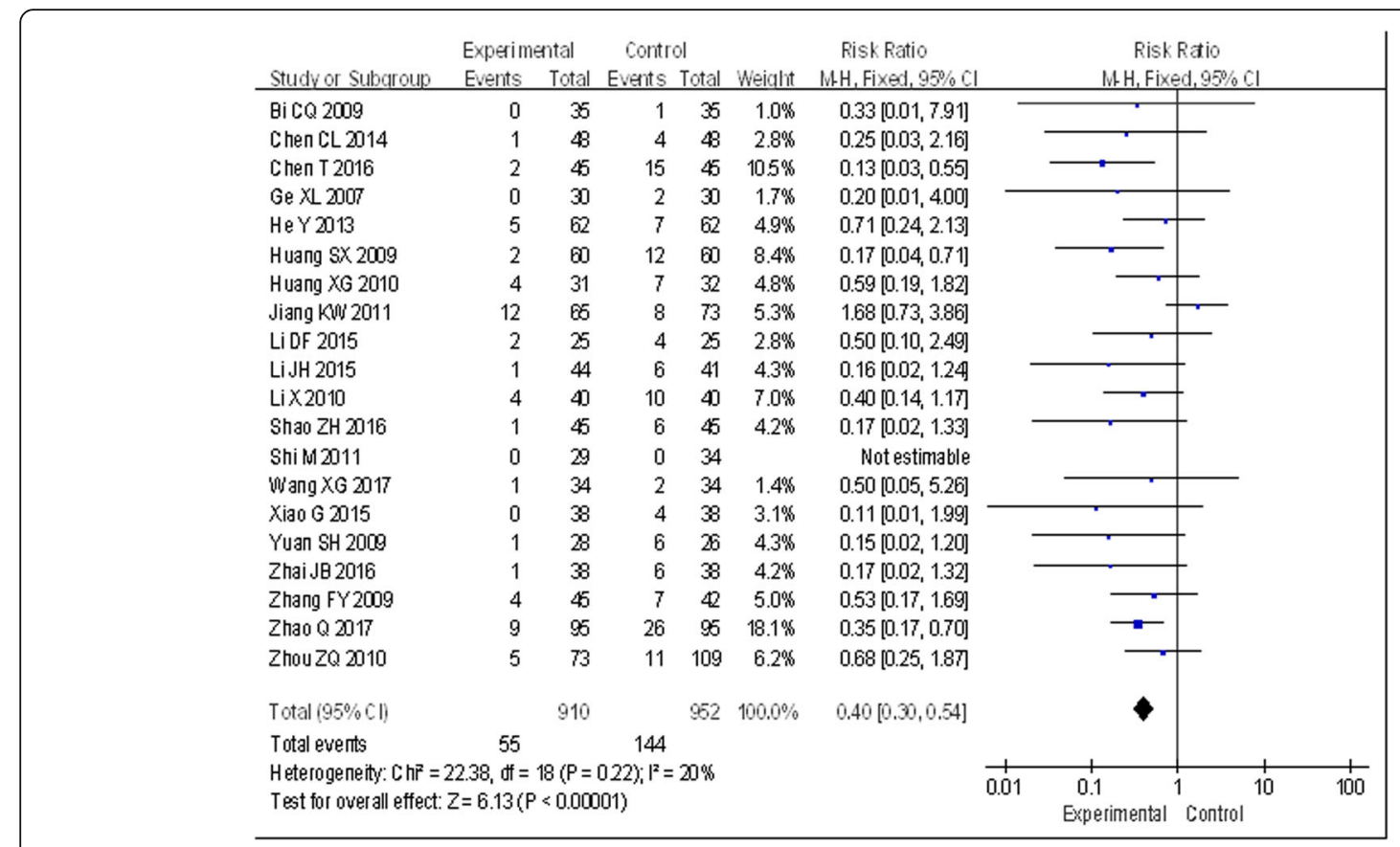

Fig. 2 Effect of traditional Chinese and western medicine on the prevention in DVT

surgery; the incidence rate of DVT was significantly lower than that of the control group with statistical difference. And the values of D-dimer was lower in the experimental group than those of the control group, with statistical difference. However, there was no statistical difference between PT and APTT in the two groups; it showed that traditional Chinese medicine did not increase the risk of bleeding or hemorrhage. Four RCTs described the incidence of subcutaneous hematoma after surgery; amazingly, meta-analysis indicated a significantly lower number of patients on the experimental group undergoing the subcutaneous hematoma compared to that in the control group, with statistical difference. In addition, there were no reports of any serious complications, demonstrating the safety of the combination of traditional Chinese and western medicine in the prevention of DVT.

Despite the lack of knowledge about the biological mechanisms of traditional Chinese medicine in the prevention of DVT, the synergy between the efficacy of Chinese herbal medicine and western medicine may play a major role in symptomatic treatment.

First, ingredients of traditional Chinese medicine can promote pain relief and flow of Qi (vital energy), reduce swelling and remove blood stasis, and bring more nutrients and oxygen to the healing tissues, so that blood circulation is improved and obstruction in the channels is removed [34]. Second, researches have suggested that

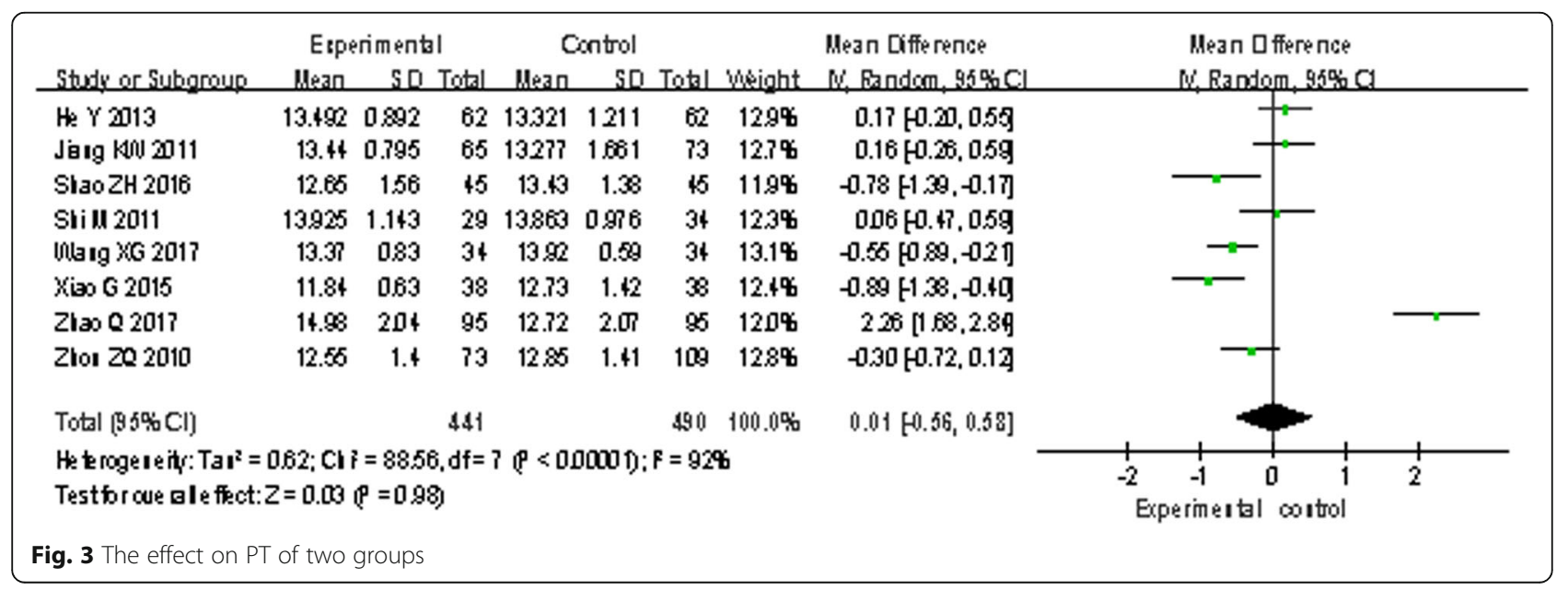




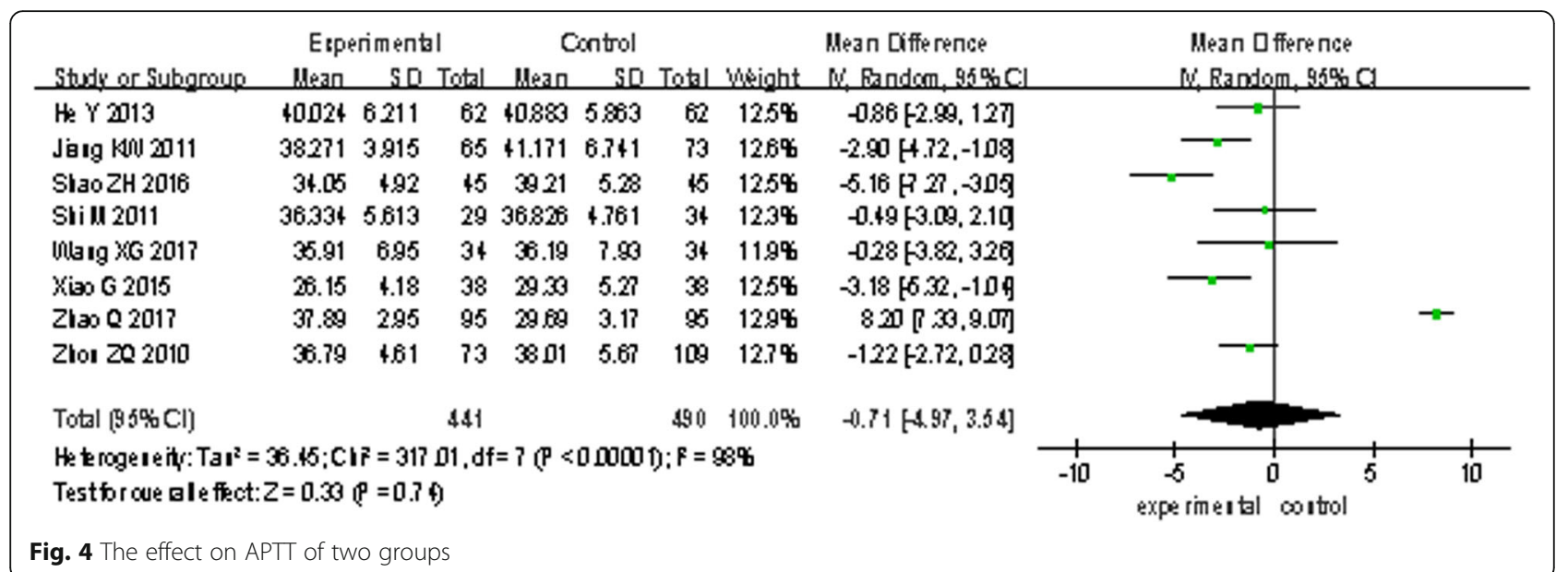

out-off-balance between the coagulation and anticoagulation system is a major reason to cause human body's hyperglycemia during the process of DVT formation. Third, traditional Chinese medicine theory studies have suggested that the basic pathogenesis of deep venous thrombosis is blood stasis in the meridians.

Some studies have already proved that traditional Chinese medicine has therapeutic efficacy in DVT. For example, Honghua [35] injection has optimal therapeutic effect by its anti-thrombosis, anti-myocardial ischemia, microcirculation improvement, antioxidant, and other aspects. Danggui [36] can expand blood vessels and inhibit platelet aggregation; besides, it also has the effect on anti-oxidation and free radical elimination. These benefits are largely dependent on its ingredients of polysaccharides, phthalates, coumarins, flavonoids, volatile oil compounds, and others. Chuanxiong [37] has optimal therapeutic effect by its bio-activity such as anti-tumor, anti-inflammation, anti-apoptosis, and vasodilation. Danshen [38] can prevent thrombosis by expanding the peripheral vessels and increasing the activity of plasmin. Some researches also indicated Danshen can exert its antithrombotic effect through inhibiting platelet aggregation (by increasing cAMP levels and inhibiting TXA2 synthesis in platelets) and improving the status of hemorheology property of the blood (by decreasing blood viscosity and shortening erythrocyte electrophoresis time).

These researches suggested that traditional Chinese medicine can eliminate obstruction in the channels for the patients with DVT by its effects on swelling alleviation, blood stasis removal, and blood circulation promotion. Cumulatively, these beneficial reports may cause the improvement of the clinical symptoms of deep venous thrombosis after lower extremity orthopedic surgery.

This is the first meta-analysis to evaluate the efficacy and safety of traditional Chinese and western medicine for the prevention of DVT after lower extremity orthopedic surgery, and all researches are RCTs to ensure the scientific reliability and rigor. But there are some limitations. First, the overall methodological quality of the RCTs was moderate. Many of the trials selected for

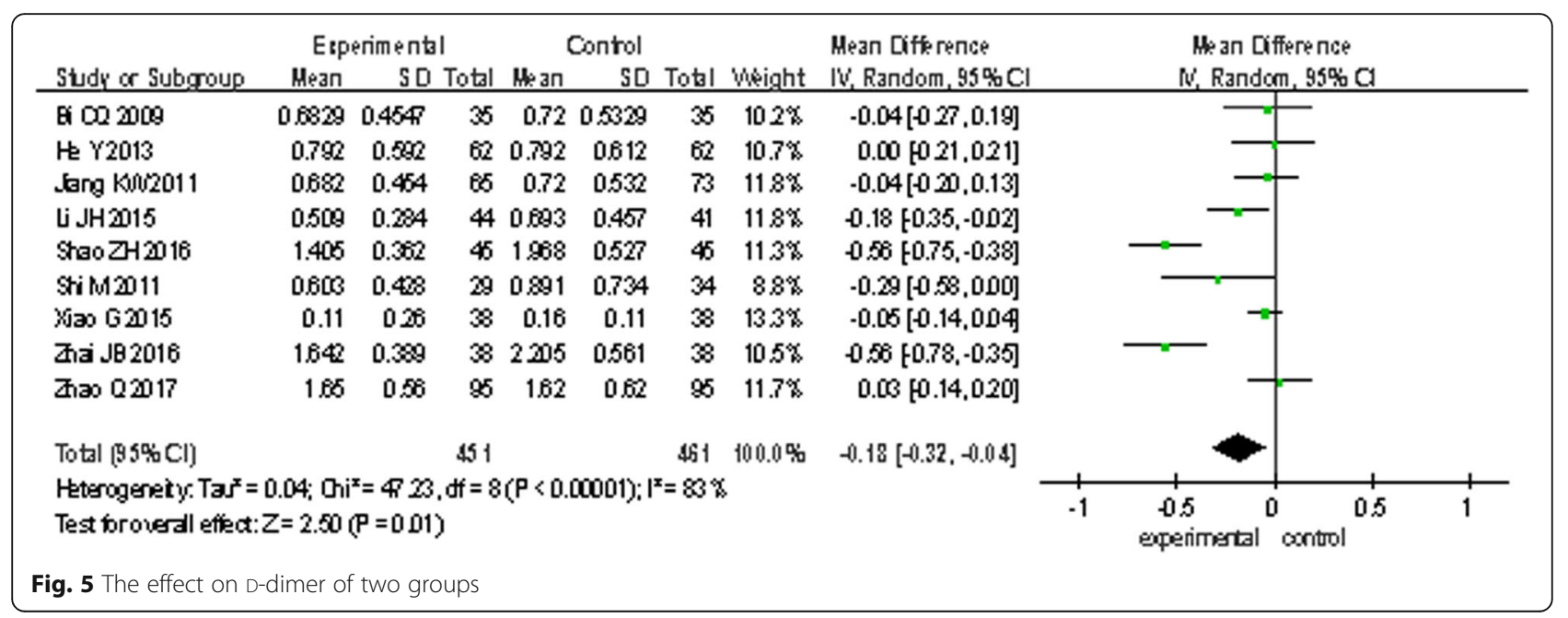




\begin{tabular}{|c|c|c|c|c|c|c|c|c|c|c|}
\hline Study or Subqroup & \multicolumn{2}{|c|}{ Experimental } & \multicolumn{2}{|c|}{ Control } & Weight & $\begin{array}{c}\text { Risk Ratio } \\
\text { M-H, Fixed, 95\% Cl }\end{array}$ & \multicolumn{4}{|c|}{$\begin{array}{c}\text { Risk Ratio } \\
\text { M-H, Fixed, } 95 \% \mathrm{Cl}\end{array}$} \\
\hline Bi CQ 2009 & 10 & 35 & 26 & 35 & $54.7 \%$ & $0.38[0.22,0.67]$ & & & & \\
\hline Jiang KW 2011 & 1 & 65 & 8 & 45 & $19.9 \%$ & $0.09[0.01,0.67]$ & & & & \\
\hline Li DF 2015 & 4 & 25 & 11 & 25 & $23.2 \%$ & $0.36[0.13,0.99]$ & & & & \\
\hline Li JH 2015 & 2 & 44 & 1 & 41 & $2.2 \%$ & $1.86[0.18,19.79]$ & & & & \\
\hline Total $(95 \% \mathrm{Cl})$ & & 169 & & 146 & $100.0 \%$ & $0.35[0.22,0.56]$ & & & & \\
\hline Total events & 17 & & 46 & & & & & & & \\
\hline $\begin{array}{l}\text { Heterogeneity: } \mathrm{Chi}^{2} \\
\text { Test for overall effec }\end{array}$ & $\begin{array}{l}3.82, d f= \\
Z=4.43(f\end{array}$ & $\begin{array}{l}(P=0 . \\
<0.00\end{array}$ & $\begin{array}{l}.28) ;\left.\right|^{2}=2 \\
001)\end{array}$ & $1 \%$ & & & 0.005 & $\begin{array}{cc}0.1 & 1 \\
\text { experimental }\end{array}$ & control $^{10}$ & 200 \\
\hline
\end{tabular}

inclusion contained some methodological deficiencies, so the number of truly high-quality studies eligible under these standards was too small. This might have caused bias. In the final results, some of the studies mentioned the word "random" but did not describe the specific method employed and did not mention whether a blind methodology was used or whether it had any dropout or not, which might cause a certain degree of bias risk. Second, all RCTs are unanimously published in Chinese academic journals due to the particularity of study contents, so we did not use statistical methods to test for publication bias. Third, some studies did not use the recommended guidelines for the prevention of DVT, and there is no explicit mention that if they conducted the evaluation of DVT after 11- to 35-day anticoagulant therapy in accordance with the guidelines. Besides, the definite location of thrombosis and whether there is some bleeding and other side effects were not mentioned. Therefore, we still need more large sample, highquality, and multi-center RCTs to support the efficacy and safety of traditional Chinese and western medicine for the prevention of DVT after lower extremity orthopedic surgery.

\section{Conclusion}

Traditional Chinese medicine have the ability of swelling reduction and blood stasis removal, also can promote blood circulation to remove obstruction in the channels for patients. Cumulatively, these beneficial reports may result in reducing the incidence rate of DVT after lower extremity orthopedic surgery. Despite moderate quality of trials included and the existed bias of researches, Chinese traditional medicine therapy with a history dating back thousands of years radiates a glimmer of hope in the prevention of DVT after lower extremity orthopedic surgery. However, more high-quality, rigorously designed, and well-controlled RCTs are needed to support the clinical application of traditional Chinese medicine for the prevention of DVT.

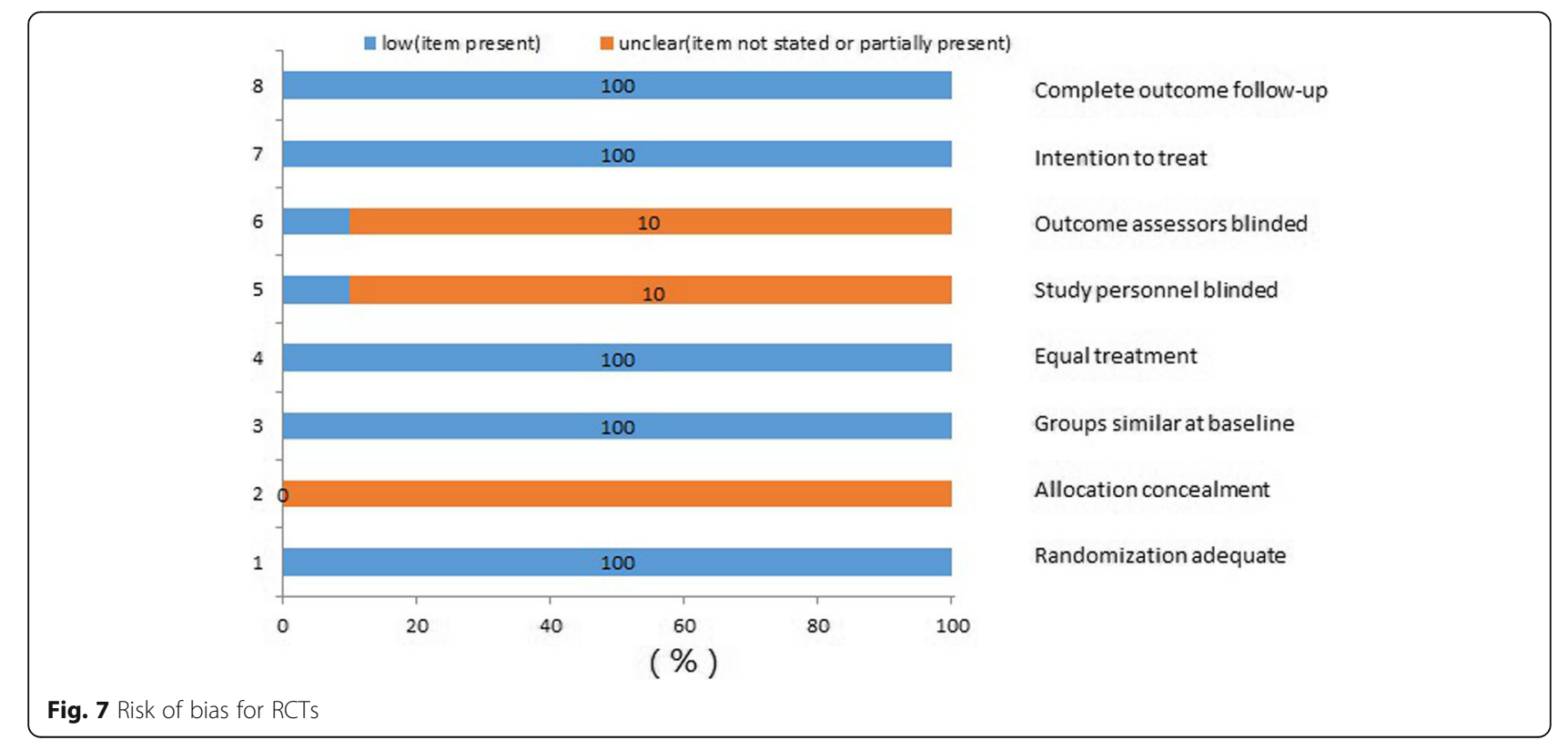




\section{Abbreviations}

APTT: Thromboplastin time; DVT: Deep venous thrombosis; GCS: Graduated compression stocking; HFS: Hip fracture surgery; IPC: Intermittent pneumatic compression devices; LMWH: Low molecular weight heparin; PE: Pulmonary embolism; PT: Prothrombin time; TCM: Traditional Chinese medicine; THR: Total hip replacement; TKR: Total knee replacement; VFP: Venous foot pump; VTE: Venous thromboembolism

\section{Acknowledgements}

We thank the teachers of Department of Orthopedics, Beijing Union Medical College Hospital, for giving advice on the article design and thank He Hongmei and Guo Xiangqian of Henan University School of Medicine for providing guidance on the design and statistical analysis.

\section{Funding}

This paper is funded by National Natural Science Foundation of China. Name: 3D-Printed Paramagnetic Titanium Alloy Scaffolds in the Repair of Osteonecrosis of Femoral Head: Insights of its mechanism and efficacy, Grant Number: 81572110.

\section{Availability of data and materials}

All supporting data can be seen in the tables.

\section{Authors' contributions}

ZS designed the study and developed the retrieve strategy. SY and CX performed the systematic evaluation as the first and second reviewers, searching and screening the summaries and titles, assessing the inclusion and exclusion criteria, generating data collection forms and extracting data, and evaluating the quality of the study. Professor QW advised and revised the inclusion/exclusion criteria. ZS and SY performed the meta-analysis of continuous and skewed results. ZS drafted the article. All authors read and approved the final draft.

\section{Authors' information}

Shibai Zhu (male, 2 December 1991) and Xi Chen (male, 7 October 1992) are medical postgraduates from the Department of Orthopedic Surgery, Peking Union Medical College Hospital, Peking Union Medical College, Chinese Academy of Medical Science, Beijing, China. Yi Song (female, 1991.1.28) is a medical postgraduate from the Department of Endocrinology and Metabolism, The First Affiliated Hospital of Zhengzhou University, Zhengzhou, Henan Province, China. Wenwei Qian (male, 19 July 1972) is a doctor of medicine, professor, from the Department of Orthopedic Surgery, Peking Union Medical College Hospital, Peking Union Medical College, Chinese Academy of Medical Science, Beijing, China.

\section{Ethics approval and consent to participate}

This is a meta-analysis; no relative problems exist.

\section{Competing interests}

The authors declare that they have no competing interests.

\section{Publisher's Note}

Springer Nature remains neutral with regard to jurisdictional claims in published maps and institutional affiliations.

\section{Author details}

'Department of Orthopedic Surgery, Peking Union Medical College Hospital, Peking Union Medical College, Chinese Academy of Medical Science, Beijing, China. ${ }^{2}$ Department of Endocrinology and Metabolism, The First Affiliated Hospital of Zhengzhou University, Zhengzhou, Henan Province, China.

Received: 31 January 2018 Accepted: 25 March 2018

Published online: 10 April 2018

\section{References}

1. Geerts $W H$, Bergqvist $D$, Pineo GF et al. Prevention of venous thromboembolism: American College of Chest Physicians evidence-based clinical practice guidelines (8th edition)[J]. Chest, 2008, 133 (6 Suppl): 381S-453S

2. Jacobs JJ, Mont MA, Bozic KJ, et al. American Academy of Orthopaedic Surgeons clinical practice guideline on: preventing venous thromboembolic disease in patients undergoing elective hip and knee arthroplasty. J Bone Joint Surg Am. 2012;94(8):746-7.

3. Qiu GX. Chinese major orthopedic surgery of venous thromboembolism prevention guidelines. Chinese J Orthopaedics. 2016:36(2):70-2.

4. Schluter J, Scotter $\mathrm{H}$, Chaboyer W. Best practice guidelines for Australia and New Zealand on the prevention of venous thromboembolism: issues and implications for nurses. Contemp Nurse. 2008;29(1):3-7.

5. Bang SM, Jang MJ, Oh D, et al. Korean guidelines for the prevention of venous thromboembolism. J Korean Med Sci. 2010;25(11):1553-9.

6. Falck-Ytter Y, Francis CW, Johanson NA, et al. Prevention of VTE in orthopedic surgery patients: antithrombotic therapy and prevention of thrombosis, 9th ed: American College of Chest Physicians evidence-based clinical practice guidelines. Chest. 2012;141(2 Suppl):e278S-325S.

7. Zhang J. A retrospective study of promoting blood and removing stasis preventing venous thrombosis after artificial hip joint replacement[D]. Hunan University of Chinese Medicine, 2014

8. Wells GA, Shea BJ, O'Connell D, et al. The Newcastle-Ottawa scale (NOS) for assessing the quality of non-randomized studies in meta-analysis. Appl Eng Agric. 2014;18(6):727-34.

9. Higgins JP, Green S. Cochrane handbook for systematic reviews of interventions. Naunyn-Schmiedeberg's Arch Exp Pathol Pharmakol. 2011; 5(2):S38.

10. Moher D, Liberati A, Tetzlaff J, et al. Preferred reporting items for systematic reviews and meta-analyses: the PRISMA statement. Ann Intern Med. 2009; 151(4):264-70.

11. Sterne JA, Sutton AJ, loannidis JP, et al. Recommendations for examining and interpreting funnel plot asymmetry in meta-analyses of randomised controlled trials. BMJ (Clinical research ed). 2011;343(5):d4002.

12. Bi CQ, Wen JM, Jiang KW, et al. Chinese drugs for supplementing Qi and activating blood circulation in preventing deep venous thrombosis after big operations in orthopaedics and traumatology. China J Chinese Materia. 2009:34(5):625-7.

13. Chen CL, Zhou SP, Hou YZ. Prevention of deep venous thrombosis of lower extremities with traditional Chinese and western medicine after total knee arthroplasty combined. China Prac Med. 2014;9(11):190-1.

14. Chen T. Preventive effect of combined traditional Chinese medicine and western medicine on deep venous thrombosis of lower extremities after orthopedics. Chinese Baby. 2016:23:65-6.

15. Ge XL, Xu GH, Shan JB. Combination of TCM and WM prevent thrombopoiesis of deep vein after artificial joint substitution. Chin Arch Tradit Chin Med. 2017;25(11):2429-30.

16. He Y, OuYang GL, Xiao LP. Effect of Yiqi Huoxue Huayu decoction on prevention of deep venous thrombosis after artificial hip replacement. ShanXi J Tradit Chin Med. 2013;34(11):1498-9.

17. Huang SX. Prevention of 60 cases of deep vein thrombosis after orthopedic surgery with salvia miltiorrhiza injection combined with low molecular weight heparin. Guangxi Med J. 2009:31(12):1787-8.

18. Huang SX. Prevention of deep venous thrombosis after artificial joint replacement in lower limbs by traditional Chinese and western medicines. CJGMCM. 2010;25(7):1264-6.

19. Jiang KW, Wen JM, Chen T, et al. Clinical observation of preventing deep vein thrombosis post major orthopedic surgery with Chinese herb. Modern I Integrated Traditional Chinese Western Med. 2011;20(12):1431-3.

20. Li DF, Yuan ZX. Clinical observation on prevention of deep venous thrombosis after orthopedic surgery by traditional Chinese and western medicine. Med Inf. 2015;28(49):312

21. Li JH, Huang ZM, Li MC. Prevention of deep venous thrombosis following hip fracture surgery with rehabilitation therapy and Dang gui Sha yao herbal powder. Chin J Surg Integrated Traditional Western Med. 2015;21(6):567-70.

22. Lin $X$, Wang RM, Guo YB, et al. Prevention of deep venous thrombosis after total hip arthroplasty with buyanghuanwu soup. J Shanxi College Traditional Chin Med. 2010;11(2):26-7.

23. Shao ZH, Zhang YF, Xu YG, et al. Effect of BushenYiqiHuoxue method on prevention of deep venous thrombosis after elderly hip arthroplasty. Modern J Integrated Traditional Chinese and Western Med. 2016;25(7):765-7.

24. Si M, Yang YM, Xu HY, et al. Effect of YiqiHuoxue method on hemorheology after artificial hip replacement. Modern J Integrated Traditional Chin Western Med. 2011;20(13):1593-4.

25. Wang XG. Clinical observation on prevention of deep venous thrombosis after orthopedic surgery by TongmaiQiqi. Shanxi J Tradit Chin Med. 2007; 38(1):72-3. 
26. Xiao G. Effect of combination of traditional Chinese and western medicine on prethrombotic state after surgery in lower limb fractures, strait. Pharmaceutical J. 2015;27(7):131-2.

27. Yuan SH, Tong PJ. Clinical study on prevention of deep venous thrombosis after total knee replacement by integrated traditional Chinese and western medicine. Beijing J Tradit Chin Med. 2009;28(2):119-20.

28. Zhai JB. Clinical effect of traditional Chinese and western medicine on prevention of deep venous thrombosis after elderly hip arthroplasty. Chin J Geriatric Care. 2016;14(5):16-8.

29. Zhang FY, Chen BG, Hou N. Prevention and treatment of deep venous thrombosis of lower limbs by traditional Chinese and western medicines. Clin Med. 2009;29(8):111-2.

30. Zhao Q, Li ZG, Li BG, et al. The effect of integrated traditional Chinese and western medicine on prevention and treatment of deep venous thrombosis after orthopedics surgery. Hebei Med J. 2014;39(7):1034-7.

31. Zhou ZQ, Liu M, Zhao YL, et al. Effect of Honghua injection on preventing deep venous thrombosis of lower extremities after orthopedics[C]. Chinese academic communication of traditional Chinese and western medicine in peripheral vascular diseases. 2010

32. Simiao S. Beiji Qianjin Yao Fang [M]. Beijing: People's Medical Publishing House; 1955.

33. Tang ZH. Xuezheng Lun[M]. Beijing: People's Medical Publishing House; 1990.

34. Zhou T, Sun DQ, Wu P, et al. Clinical research on Sanmiao powder combined fibrinogenase for injection in treatment of acute deep venous thrombosis of lower limbs. CJTWM. 2012;32(7):918-21.

35. Wei GF, Xu L, Yao H. Identification criteria and pharmacological research of Honghua. Shanxi J Traditional Chin Med. 2010;31(6):736-7.

36. Liu YH, Yang SY, Ma WL, et al. Research progress of pharmacological action of Danggui. China Modern Med. 2014;21(22):192-4. 196

37. Wu TT, Ding YY. The recent research review of bioactivity of Ligustrazine. Food Res Dev. 2016;37(6):212-7.

38. Feng J, Wang SC. Effect of Fufang Danshen Diwan to platelet aggregation function. Chin J Misdiagn. 2006;6(12):2261-3.

\section{Submit your next manuscript to BioMed Central and we will help you at every step:}

- We accept pre-submission inquiries

- Our selector tool helps you to find the most relevant journal

- We provide round the clock customer support

- Convenient online submission

- Thorough peer review

- Inclusion in PubMed and all major indexing services

- Maximum visibility for your research

Submit your manuscript at www.biomedcentral.com/submit 